Review

\title{
Gut Microbiome Production and Modulation of Hormones That Influence Host Skin Health
}

\author{
Mia Maguire* and Greg Maguire \\ NeoGenesis Inc \\ San Diego, CA USA \\ ${ }^{*}$ Corresponding author \\ maguiremm@yahoo.com
}

\begin{abstract}
Recently scientific research began to shift their focus on looking at both the gut and the skin microbiota as having a reciprocal and integral relationship with one another, rather than assessing them as separate and unrelated fields. In the past five years, the field of microbial endocrinology emerged, which examines how our gut microbiota influences and modulates hormones. We've known for decades that hormones greatly affect the condition of the skin, and many skin conditions are often treated with oral hormonal therapy as means to internally treat skin conditions visible on the dermis. Now, a growing body research and discourse examining this triad of biological spheres - gut microbiota, skin microbiota, and the endocrine system - as interconnected rather than binary and unrelated. While there is ample research established and being conducted examining the gut-skin axis, the gut-brain axis, and the gut-hormone axis, through this paper I will review and synthesize some of the significant advancements in this emerging and inclusive field of science to suggest that the fields need to expand the axis and their modality for researching these fields as a connected whole in order to better understand the role of the microbiota in disease prevention as a whole.
\end{abstract}

\section{Introduction}

The gut microbiota may be contiguous with the microbiome of other parts of the body, and is a key part of our innate and adaptive immune systems (Maguire and Maguire, 2017). Performing a number of other essential functions, such as protective, structural, and metabolic functions, the gut's microbiome is key to our health. Indeed, without a gut microbiome, as in gnotobiotic mice, where host health no longer benefits from the microbiota's food processing, digestion of complex host-indigestible polysaccharides, pathogen displacement, and synthesis of vitamins, neurotransmitters and hormones, the health of the host deteriorates ( $\mathrm{Lu}$ et al, 2018). In animal models a depleted gut microbiome, gnotobiotic mice for example, can lead to many diseases including Parkinson's Disease, and in the skin, malformed coenocytes have been described (Iizuka et al, 2009). 
In this review, I'll focus on how the gut's microbiome produces and modulates hormones that in turn influence the skin of the host. This is an emerging field of study, and no reviews have been published on the topic. Although I focus on the gut influencing the skin, we must also recognize the skin itself as an endocrine organ given that skin synthesizes vitamin D and sex steroids for example (Zouboulis, 2009), and that the skin's microbiome plays an important role in skin health, and may directly affect the microbiome in other parts of the body (Maguire and Maguire, 2017). Many of the studies I review here are associative, where proper experimental controls are not in place and therefore causative conclusions cannot be made, but the data do provide ample evidence of an important subject that merits further experimental research.

Gut dysbiosis has been found to impact a number of different psychological and biological functions in the host's health, from neurotransmitter functioning to metabolism, along with being associated with a slew of different diseases and disorders from Parkinson's disease to leaky gut syndrome (Nash et al, 2017). Because gut flora plays a significant role in the metabolizing of hormones and the hormones can greatly influence the health of our skin (Langan et al, 2018), it's important for research to not only consider the gut-skin connection, but also the triangular relationship between the gut, the endocrine system, and the skin.

Research on the microbiota of the skin has skyrocketed in recent years, prompting a wide range of skincare companies to adopt probiotic and prebiotics as active ingredients in their topical treatments (Maguire and Maguire, 2017). Until now, the focus has been on topical treatment of common skin problems, from acne to fine lines and wrinkles. But as we know, the physical condition of the skin is profoundly affected by not just what is applied topically to the skin, but also by a number of different factors including lifestyle, hormones, nutritional health, and, as we will seek to demonstrate in this paper, the gut microbiota's action in producing and modulating hormones.

Hormones play a significant role in a number of different skin conditions, from acne vulgaris to melasma, but more significantly, the hormone-skin-gut connection have a tri-directional relationship in which each three affect and even modulates the other.

While sex hormones including estrogen, testosterone, and progesterone are most commonly linked with skin conditions and issues, hormonal function in the gut also modulates mood and sleep disorders including serotonin and melatonin, stress hormones including cortisol, the thyroid hormones T3 and T4, and insulin levels that will also affect the skin.

An unhealthy gut microbiota can alter the normal functioning and balance of all of these hormones, and such imbalances internally can present themselves externally by way of the epidermis. Patients with undesired skin conditions often seek the advice of a dermatologist before an endocrinologist, or even a primary care physician, and this is problematic because the field of dermatology, as well as western medicine and diagnostic assessing as a whole, has been conceived of and maintained with a binary, onedirectional approach to both diagnostic methodology as well as treatment plans for decades. However, in recent years the importance of studying the gut-hormone-skin relationship has come to surface not only in scientific research, but is slowly beginning to shift the ways in which practitioners think about illness and skin conditions (Vaughn et al, 2017). 
As a result of the growing body of research of the gut's many implications on overall health and wellness, there has been a shift towards a more multidimensional approach to looking at illness and health conditions (Bowe and Logan, 2011), with a growing focus on how gut health and functioning is inevitably a major factor in not only hormonal functioning as a whole, but hormonal-related skin issues like acne, melasma, rashes, and other epidermal conditions not explained by other factors (Parodi et al, 2008).

\section{ESTROGEN}

In general, acne is thought to be caused by an overgrowth of bacteria on the host's pores, with an increased incidence of Propionibacterium acnes biofilms (Jahns et al, 2012). But this bacterial imbalance isn't typically the result of poor hygiene or failure to properly cleanse the skin. In fact, the skin's condition isn't isolated to what goes on the epidermal level altogether. Acne is a form of inflammation, and we know that both the functioning of the gut microbiota as well as our hormones, have a direct relationship with inflammatory responses both internally and externally (Kim et al, 2017). Interestingly, Sanford et al (2016) have shown that $P$. acnes when trapped in sebum will ferment the sebum into inflammatory short chain fatty acids (SCFSs). The acne breakouts in teenagers and menstruating women may therefore be, at least partially, a consequence of the hormonal controlled increase in sebum production (Pochi, 1990) and resulting inflammatory SCFAs.

For instance, many dermatologists often prescribe oral contraceptives containing the hormone estrogen to patients with cystic acne who have not responded to topical treatments such as salicylic acid, benzoyl peroxide, and even prescription retinoids. Using estrogen-based contraceptives is often an effective treatment for women suffering from cystic acne as estrogen is known for its oil-reducing and beneficial properties (Thorton, 2005). However, too much estrogen can also lead to exacerbated acne as well. It is believed that contraceptives work to treat acne by balancing out levels of progesterone and estrogen, and thus reducing the excess production of acne-causing sebum.

However, recent studies have begun to illuminate the interplay between skin health and the endocrine system is far more complex than simply causing elevated levels of oil. Commensal bacteria can both modulate and secrete hormones (Neuman et. al, 2015). In one study two estrogen-derivative sex hormones, estriol and estradiol, were found to decrease the bacterial virulence of the species Agrobacterium tumefaciens and P. aeruginosa (Roshchina, 2010). Quorom-sensing is where bacterial populations synthesize and exchange chemical signals that coordinate and synchronize gene expression. The host derived hormones estriol and estradiol have been shown to inhibit quorum-sensing and thus decrease bacterial virulence (Beury-Cirou et al, 2013).

Furthermore, estrogen has been found to regulate glucose, lipid metabolism and inflammatory responses. Another hormone associated with acne and oil production is testosterone. Elevated levels of testosterone are often caused by resistance to insulin, and in turn to increased oil production, which can lead to acne. Abnormally elevated insulin can be associated with gut dysbiosis when the dysbiosis is triggered by a high fat diet (He et al, 2018).

Melasma is another skin condition causing hyperpigmentation on the skin that's been linked with gut dysbiosis, and more specifically with leaky gut syndrome. Common in pregnant women and women taking 
oral contraceptives, melasma typically presents itself in the form of brown spotting or patches on the chin, forehead, and cheeks in a symmetrical formation (Sheth and Pandya, 2011). Melasma occurs when there is excess melanin production, and can also be triggered by of a number of different environmental and physiological factors, from UVR exposure to hormones and inflammatory signaling. Gut microbiota changes profoundly during pregnancy from the first to third trimester. A recent study that sampled the fecal bacteria of a 91 pregnant mothers and their infants, found a distinct shift in overall increase in Proteobacteria and Actinobacteria from the first to the third trimester and overall increase in inflammation (Koren et. al, 2012). During pregnancy, both inflammatory and anti-inflammatory responses are altered to protect against fetal rejection and inhibit the transfer of maternal antibodies to the fetus (Robinson and Kelin, 2012). These inflammatory immune signals are modulated by the increase of estrogens and steroid hormones throughout the pregnancy, including estradiol, estriol, progesterone, and glucocorticoids. Hence, the change in inflammatory immune signaling by a change in female sex hormones can be connected to the onset of melasma and hyper-pigmentation in women with increased estrogen.

Another symptom of hormonal changes in women that can occur during both pregnancy and menopause is gastroesophageal reflux (GERD). Symptoms of GERD include heartburn and acid reflux and this condition is often the cause of or triggered by disruptions in the production of stomach acids. While GERD affects approximately $60 \%$ of the population at least once per year (Jacobson et. al, 2008), and can be caused by dietary and lifestyle choices, a lack of the production of stomach acids is often the culprit whether triggered by hormonal or dietary influences. Other symptoms of GERD are not only associated acid reflux, bloating, and digestion issues, and redness in the skin. A study looking at the connection between hormones and GERD in postmenopausal women found that GERD symptoms were significantly increased when estrogen (symptoms among postmenopausal women using $\mathrm{PMH}$, selective estrogen receptor modulators, or over-the-counter hormones) dosage or duration of treatment increased (Jacobson et. al, 2008).

While the study didn't look at the effects of the estrogen-induced GERD implications to the skin, pregnant women often reports red, blotchy, itchy, and unusually sensitive skin. It is plausible that this same irritation may be linked with not only a direct change in estrogen and progesterone, but could also be the result of stomach acid dysfunction, GERD, and other gut-related issues associated endocrinal activity.

\section{OXYTOCIN \& HPA HORMONES}

Oxytocin is a hormone that acts as neurotransmitter in the brain, and is associated with maternal-infant bonding, empathy, orgasm, and feelings of kindness, leading to its nickname as the "love hormone." While it's often thought of as an exclusively female hormone, oxytocin actually affects testosterone production in men.

A recent study found that probiotic-treated mice not only displayed heightened social grooming behaviors, but also displayed "more radiant skin," when compared to mice who were not treated with probiotics (Erdman and Poutahidis, 2014). In the "glow of health" study, researchers found that mice who were treated with Lactobacillus reuteri presented upregulated levels of IL-10, which is an anti-inflammatory cytokine proposed to regulate the hypothalamic-pituitary-adrenal (HPA), axis hormones - including 
oxytocin - both indirectly and directly (Roque et al., 2009). This interfacing may affect the skin because IL10 reduces proinflammatory cytokines including IL-17. Reduced levels of IL-17 have been shown to promote skin-enhancing benefits such as increased hair follicle number and dermal thickness (Erdman and Poutahidis, 2014).

Another recent study in 2013 also found oxytocin to be an integral factor in the multi-directional gut microbe-brain-immune axis by assessing the role of probiotic treatment and its effect on wound healing in (Poutahidis et al., 2013). This study found that female mice who were treated with dietary Lactobacillus reuteri (L. reuteri) displayed an increase in social grooming when compared to the control, and showed better rates in wound healing as a result. There are several factors that impact the wound healing process, ranging from collagen deposition to inflammation. Both of these factors are not only important for wound healing, but they're also associated with aging of the skin. Decreased collagen levels along with inflammation can lead to an increase in premature signs of aging, from loss of elasticity to fine lines. The same study looked closely at the relationship between collagen depletion and deposition and the impact that the probiotic treatments may have had in the sample. The researchers found that $w t$ mice that were given the L. reuteri treatment showed an increased rate in collagen deposition in the dermis. This finding illuminates a potential connection between using probiotics as an ingestible treatment for aging, which may be more effective than consuming collagen supplements that are currently marketed for cosmetic purposes.

\section{LEAKY GUT SYNDROM, HORMONES, AND SKIN}

Aside from excessive fatigue, memory loss, and gastrointestinal symptoms include constipation and chronic diarrhea as a result of inflammation in the intestinal walls, one of the most common symptoms of leaky gut syndrome may be skin rashes (Pietrzak et al, 2017). These rashes associated with leaky gut and be the result of the body's immune response in its attempt to rid the body of excessive toxin being released from the bloodstream into the skin (Bowe and Logan, 2011). Leaky gut syndrome has also been commonly comorbid with cystic acne and psoriasis, which have had a long term association with hormonal imbalances (Ceovic et al, 2013), leading many dermatologists and endocrinologist to prescribe oral contraceptives to patients not as a form of birth control, but as an acne treatment. While contraceptives have been effective treatment for many women in combatting cystic acne for decades, recent studies assessing the role of the gut microbiota and skin have shown that the causality of hormonal acne may be much more complex and further, have illuminated that hormonal imbalances that adversely affect the skin, may be improved by restoring balance to the gut through diet and probiotic supplementation (Mottin and Suyenaga, 2018).

Estrogen metabolites stay in circulation in the gut, which can cause estrogen dominance, and thereby disrupt the normal balancing act of progesterone and estrogen. As mentioned, estrogen is perhaps the most significant hormone when it comes to assessing skin health related to hormonal triggers. This connection is an important consideration when looking at the relationship between sex hormones and the gut microbiota because it brings forth the idea that healing hormonal imbalances which - among other things 
- affect the health of the skin may be an effective alternative treatment to traditional hormone replacement and oral contraceptives therapies.

While incorporating a probiotic and/or prebiotic supplement into a one's diet may not be sufficient enough to improve the skin or reduce dermatologic disorders alone, changing a diet to include more probiotics, prebiotics, and fermented foods as a way of healing the gut microbiota. In turn, restoring a functional hormonal balance in the endocrine system may be considered a primary course of treatment for dermatologists and primary care physicians to consider prior to resolving to prescribing prescription medications, including oral contraceptives and the acne medication Accutane, known for causing a number of different side effects.

\section{ANTIBIOTICS, HORMONES, AND SKIN}

While oral antibiotics are often a necessary course of treatment for a wide range of infections and infectious diseases, the overuse of them has proven to be detrimental to the gut microbiota as a whole, and has also proven to be a contributing factor to numerous gastrointestinal diseases, including leaky gut syndrome and a weakened immune system. In addition to the rampant over prescribing of antibiotics to patients, we are also increasingly ingesting more hormones and antibiotics through the food we eat more than ever before (Jeong et. Al, 2010).

Both antibiotics and hormones are used legally (and illegally) in food production to promote faster growth in livestock (Jeong et. Al, 2010). Some of the hormones used for these purposes include estradiol-17 $\beta$, progesterone, testosterone, and synthetic hormones including ADIs and MRLs. As mentioned before, an imbalance in such hormones can disrupt the functioning of the microbiota and can lead to gut-related conditions due to inadvertently consuming excess hormones through the intestines. In this way, just like taking oral contraceptives or hormonal replacement drugs, simply by consuming dairy and meat we are in turn, consuming hormones, steroids, and antibiotics.

Researchers are beginning to examine the relationship between increased consumption of growth hormones and antibiotics through the food we consume, and the effect of how this dietary shift influences the endocrine system. This investigation is in part due to the fact that young females are reaching puberty and beginning their menstrual cycles earlier than ever before in recent history. Beginning in the 1990s, the average age of menarche saw a large spike at earlier ages in females in the United States, and today the average age of menarche begins before the age of 12 with recent studies indicating the age is declining even further (Forman et al, 2013). While the cause of the decline in the onset of puberty in U.S. females is undoubtedly a multi-faceted issue with myriad of different causes, ample research has linked this trend to ---at least in part---the increase consumption of gut-altering hormones ingested via the food we eat.

While the increased ingestion of hormones can cause a disruption in the normal balance and regulation of both female sex hormones and stress hormones, such imbalances in the endocrine system are profoundly linked to skin condition and dermatologic issues like acne at a younger age. While this connection may seem binary, the problem is that when women are prescribed hormonal oral contraceptive or other hormone modulation medications from an earlier age, this artificially "balances," the hormones in the woman and when she has to stop taking the contraceptives due to pregnancy, age, and other reasons, the 
hormones have a difficulty re-regulating themselves without the assistance of hormonal therapy. This may account for the recent spike in acne vulgaris in adult women (Zeichner et. Al, 2013). In a recent photo-based study that surveyed a total of 2,895 females ranging from the age of 10 to 70 , approximately $55 \%$ reported experiencing some form of acne. While the study acknowledged that it is unclear whether or not the spike in acne-related visits to dermatologists is due to heightened awareness of the condition or truly an increase in those suffering from the dermatologic condition, the researchers did find that there was a higher prevalence of hormonal issues and conditions such as PCOS in the adult women who came sought help for acne (Zeichner et. Al, 2013).

\section{PCOS}

While these shifts in hormones and skin conditions and diseases still need more research to confirm a bidirectional and clear relationship, it should be noted that PCOS -another hormonal disease that's seen a spike in numbers in the last decade - also has a connection to diet and alternations in the patient's microbiome. In fact, there a growing body of research that shows that PCOS (Polycystic Ovary Syndrome) can be treated - or at least symptoms of the disease can be lessened - by making changes in the patient's diet.

PCOS (Polycystic Ovary Syndrome) is an endocrine disorder that affects nearly 5 million women of childbearing age in the U.S (Ndefo et al, 2013). While the research pinpointing the exact cause of the disorder is still murky, it is generally accepted that disorder tends to stem from an excessive amount of androgens (male sex hormones) in females. Some of the symptoms of the disorder include infertility, irregular or heavier menstrual cycles, mood disorders, and acne. Again, oral contraceptives containing estradiol are often the first treatment that is prescribed to help alleviate the symptoms of the disorder with the intention of balancing out androgens with a dose of female sex hormones, along with hormoneregulating prescription drugs including testosterone-blockers such as DHT.

However, while research remains inconclusive, there is a growing body research indicating that altering one's diet may help alleviate or improve symptoms of those suffering with PCOS (Msir et al, 2016), which may shed more light on the endocrinal-gut-skin relationship as a whole. While more research is needed on this emerging field, anecdotal success stories touting the benefits of dietary changes and the incorporation of probiotics and prebiotics in patients have prompted some gynecologists to suggest dietary treatment prior to resorting to more intensive prescription drugs (Karamali et al, 2018).

\section{HORMONES, COLLAGEN, AND THE GUT}

The third major subcutaneous layer of our skin, the hypodermis is the layer beneath the skin's surface comprised of adipocytes that store fat. This is also the layer in which collagen and elastin attach. Collagen is used both orally and topically as an anti-aging supplement, thought to promote firmness to the skin and thus soften fine lines, but it's also connected to hormonal and proper gut functioning as well. Collagen is found in amino acids (arginine, glutamine, glycine and proline), which together, help to maintain balanced levels of hormones in the endocrine system. Most significantly, collagen is especially prominent in modulating the stress hormone cortisol and the sex hormone estrogen. For many menopausal and postmenopausal women, skin conditions such as rosacea, dehydration, and loss of firmness are common due 
to the reduction of estrogen and GAGS (Rittie, et al, 2008). Estrogen has been proven to have antiinflammatory properties, hence resulting in decreased estrogen levels promoting inflammation, which as a result can not only exacerbate a myriad of skin issues from acne to rosacea expediting the aging process of the dermis, but it can also disrupt the functioning of the gut as a whole, which can further influence the condition of the skin (Verdier-Sévrain, 2006).

\section{CONCLUSION}

While there is not only ample research established and currently being conducted on the gut-skin axis, the gut-brain axis, and the gut-hormone axis, each sector of microbiota research seems to slant toward exclusive and binary modalities of assessing the binary relationship between the gut and another psychological area, rather than looking at each system of integral and reciprocal. Future studies in animal models and humans will need to include integrative approaches that measure the results of interventional protocols in all three of the axis. Such an approach is now feasible given metabolomics is a key discipline for measuring endogenous synthesis or transport rates of biomolecules such as fatty acids, cholesterol, proteins and carbohydrates and proliferation rates of cells, and that complements the other 'omics' such as genomics, transcriptomics, lipidomics, and proteomics. For example, "virtual biopsies" are possible using mass spectrometer measurements of protein turnover rates following heavy water labeling (stable isotopes) in interventional studies in humans (Hellerstein and Evans, 2017). Culture and DNA methodologies for categorizing bacterial populations in gut and skin, and elsewhere are also now well advanced. Therefore, combinatorial experimental methodologies combined with computational, for example network analysis techniques as used in toxicology (Kiani et al, 2018) may illuminate the complexities underlying the interactions of the three axis.

Now that science has established a solid understanding that the gut is closely connected to the host's overall health - and an ample body of research and clinical studies serving as the backbone- in a wide variety of different psychological aspects, researchers and medical practitioners need to be encouraged to assess their patient's conditions with broad perspective that encompasses research in all three area. This will help not only better diagnose and treat patients - as well as in some cases, perhaps eliminate the need to prescribe prescription medications in lieu of dietary changes and supplementation with pre and probiotics- but also may help physicians understand comorbid diseases of which the patient wasn't aware. Furthermore, there is also research indicating a possible connection between obesity and inflammatory skin conditions as a result of estrogen imbalances in the endocrine system (Zhang and Silverberg, 2015). This area of research could potentially serve to illuminate the relationships between body mass index, hormonal imbalances, and skin conditions.

\section{References}

Beury-Cirou A, Tannières M, Minard C, Soulère L, Rasamiravaka T, Dodd RH, Queneau Y, Dessaux Y, Guillou C, Vandeputte OM, Faure D. (2013) At a supra-physiological concentration, human sexual hormones act as quorum-sensing inhibitors. PLoS One. 8(12):e83564. doi: 10.1371/journal.pone.0083564. eCollection 2013. 
Biro FM, Khoury P, Morrison JA: Influence of obesity on timing of puberty. Int J Androl 2006;29:272-277; discussion 286-290.

Bowe W and Logan A (2011) Acne vulgaris, probiotics and the gut-brain-skin axis - back to the future? Gut Pathog. 3: 1.

Ceovic R et al (2013) Psoriasis: Female Skin Changes in Various Hormonal Stages throughout Life - Puberty, Pregnancy, and Menopause. BioMed Research International Volume 2013, Article ID 571912

Erdman SE and Poutahidis T (2014). Probiotic 'glow of health': it's more than skin deep Benef Microbes. 2014 Jun 1;5(2):109-19. doi: 10.3920/BM2013.0042.

Forman MR et al (2013) Life-course origins of the ages at menarche and menopause. Adolesc Health Med Ther. 4: 1-21.

He C et al (2018) High-Fat Diet Induces Dysbiosis of Gastric Microbiota Prior to Gut Microbiota in Association With Metabolic Disorders in Mice. Front Microbiol. 2018; 9: 639.

Hellerstein M and Evans W (2017) Recent advances for measurement of protein synthesis rates, use of the 'Virtual Biopsy' approach, and measurement of muscle mass. Curr Opin Clin Nutr Metab Care. 20(3):191200. doi: 10.1097/MCO.0000000000000370.

lizuka R, Kawakami K, Chiba K. (2009) Gut bacteria producing phenols disturb keratinocyte differentiation in human skin. Microbial Ecology in Health and Disease 21:3-4, pages 221-227.

Jacobson, B. C., Moy, B., Colditz, G. A., \& Fuchs, C. S. (2008). Postmenopausal hormone use and symptoms of gastroesophageal reflux. Archives of Internal Medicine, 168(16), 1798-1804.

Jahns AC, Lundskog B, Ganceviciene R, Palmer RH, Golovleva I, Zouboulis CC, McDowell A, Patrick S, Alexeyev OA (2012) An increased incidence of Propionibacterium acnes biofilms in acne vulgaris: a casecontrol study. Br J Dermatol. 2012 Jul;167(1):50-8. doi: 10.1111/j.1365-2133.2012.10897.x. Epub 2012 Jun 1.

Karamali M et al (2018) Effects of Probiotic Supplementation on Hormonal Profiles, Biomarkers of Inflammation and Oxidative Stress in Women With Polycystic Ovary Syndrome: A Randomized, DoubleBlind, Placebo-Controlled Trial. Arch Iran Med. 21(1):1-7

Kiani NA, Shang MM, Zenil H, Tegner J (2018) Predictive Systems Toxicology. Methods Mol Biol. 1800:535557. doi: 10.1007/978-1-4939-7899-1_25.

Kim $M$ et al (2017) Inflammatory bowel disease is associated with an increased risk of inflammatory skin diseases: A population-based cross-sectional study. J Am Acad Dermatol. 76(1):40-48.

Koren O et al (2012) Host remodeling of the gut microbiome and metabolic changes during pregnancy. Cell. 150(3):470-80. 
Langan EA et al (2018) Prolactin as a candidate sebotrop(h)ic hormone? Exp Dermatol. 2018 Mar 27. doi: 10.1111/exd.13545.

Lu J et al (2018) Effects of Intestinal Microbiota on Brain Development in Humanized Gnotobiotic Mice. Sci Rep. 8: 5443.

Maguire M and Maguire G (2017) The role of microbiota, and probiotics and prebiotics in skin health. Arch Dermatol Res. 309(6):411-421.

Msir A et al (2016) COMPARISON OF DIET IN WOMEN OF REPRODUCTIVE AGE WITH AND WITHOUT DIAGNOSED POLYCYSTIC OVARY SYNDROME - PILOT STUDY. Med Pregl. 2016 Sep;69(910):274-280

Mottin VHM, Suyenaga ES (2018) An approach on the potential use of probiotics in the treatment of skin conditions: acne and atopic dermatitis. Int J Dermatol. 2018 Apr 20. doi: 10.1111/ijd.13972

Nash MJ et al (2017) Early Microbes Modify Immune System Development and Metabolic HomeostasisThe "Restaurant" Hypothesis Revisited. Front Endocrinol (Lausanne). 13;8:349.

Ndefo UA et al (2013) Polycystic Ovary Syndrome. A Review of Treatment Options With a Focus on Pharmacological Approaches. Pharm Ther.. 2013 Jun; 38(6): 336-338, 348, 355.

Neuman H et al (2015) Microbial endocrinology: the interplay between the microbiota and the endocrine system. FEMS Microbiology Reviews, 39: 509-521

Parodi A et al (2008) Small Intestinal Bacterial Overgrowth in Rosacea: Clinical Effectiveness of Its Eradication. Clinical Gastroenterology and Hepatology 6: 759-764

Pietrzak D et al (2017) Digestive system in psoriasis: an update. Arch Dermatol Res. 309(9): 679-693.

Pochi PE (1990) The pathogenesis and treatment of acne. Annu Rev Med. 41:187-98.

Poutahidis T, Kearney SM, Levkovich T, Qi P, Varian BJ, Lakritz JR, et al. (2013) Microbial Symbionts Accelerate Wound Healing via the Neuropeptide Hormone Oxytocin. PLoS ONE 8(10): e78898.

Rittie, L., et al. (2008), Induction of Collagen by Estradiol: Difference Between Sun-Protected and Photodamaged Human Skin In Vivo. Arch Dermatol, 144(9): 1129-1140.

Robinson DP and Klein SL. (2012) Pregnancy and pregnancy-associated hormones alter immune responses and disease pathogenesis. Horm Behav. 62(3):263-71

Roque, S., Correia-Neves, M., Mesquita, A.R., Almeida Palha, J. and Sousa, N., 2009. Interleukin-10: a key cytokine in depression? Cardiovascular Psychiatry and Neurology 2009: 187894.

Roshchina V Lyte M Fitzgerald P (2010) Evolutionary considerations of neurotransmitters in microbial, plant, and animal cells Microbial Endocrinology: Interkingdom Signaling in Infectious Disease and Health. New York Springer 1752 
Ryoko Iizuka, Koji Kawakami, Naoki Izawa \& Katsuyoshi Chiba (2009) Phenols produced by gut bacteria affect the skin in hairless mice,Microbial Ecology in Health and Disease, 21:1, 50-56.

Sanford JA, Zhang LJ, Williams MR, Gangoiti JA, Huang CM, Gallo RL (2016) Inhibition of HDAC8 and HDAC9 by microbial short-chain fatty acids breaks immune tolerance of the epidermis to TLR ligands. Sci Immunol. 1(4). pii: eaah4609. doi: 10.1126/sciimmunol.aah4609.

Sheth, V. M., \& Pandya, A. G. (2011). Melasma: a comprehensive update: Part I. Journal of the American Academy of Dermatology, 65(4), 689-697.

Thornton MJ (2005) Oestrogen functions in skin and skin appendages. Expert Opin Ther Targets. 9:617-29. Vaughn AR et al (2017) Skin-gut axis: The relationship between intestinal bacteria and skin health. World J Dermatol. 6(4): 52-58

Verdier-Sévrain, S., et al. (2006), Biology of estrogens in skin: implications for skin aging. Experimental Dermatology, 15: 83-94.

Zhang A, Silverberg JI. (2015), Association of atopic dermatitis with being overweight and obese: a systematic review and metaanalysis. J Am Acad Dermatol. 606-16.

Zeichner JA, Baldwin HE, Cook-Bolden FE, Eichenfield LF, Friedlander SF, Rodriguez DA. (2017) Emerging Issues in Adult Female Acne. The Journal of Clinical and Aesthetic Dermatology.10(1):37-46.

Zouboulis CC (2009) The skin as an endocrine organ. Dermatoendocrinol. 1(5): 250-252. 